Forum Kind Jugend Sport 2021 - 2:79-86 https://doi.org/10.1007/s43594-021-00031-y Angenommen: 28. April 2021

Online publiziert: 15. Juni 2021

๑) Der/die Autor(en) 2021

Gerhard Treutlein

Zentrum für Dopingprävention, Pädagogische Hochschule Heidelberg, Heidelberg, Deutschland

\title{
Dopingprävention - aber warum und wie?
}

\section{Eine Hinführung zum Präventionsansatz der dsj}

Sauberkeit im Spitzensport. Gleichwohl durfte schon in den frühen Tagen des modernen Sports niemand anfangen danach zu fragen, wie Leistungen zustande kamen. Der Blick hinter die Kulissen gilt bis heute in einigen Kreisen des Spitzensports als verpönt und stößt - außer bei aufgedeckten Skandalen - meist auf Ablehnung. Wie aber sollen junge Menschen entscheidungsfähig werden, wenn sie nur mit dem angeblich so guten Ruf des Spitzensports konfrontiert werden, nicht aber auch mit dessen möglichen Schattenseiten?

\section{Leistungssport und Medizin}

Doping war vor und zu Beginn des 20. Jahrhunderts wenig verbreitet. Für die damalige Wissenschaft war der $^{*}$ die Sportler*in selbst als Untersuchungsund Beobachtungsgegenstand interessant, nicht aber die Anwendung von Forschungsergebnissen zum Erzielen von Leistungssteigerungen (Hoberman 1994, S. 17). Später wurde der Mensch im Spitzensport zum Versuchskaninchen für wissenschaftliche Neugier. Anhand von Spitzensportler*innen als Beispiele zur Ausweitung biologischer Grenzen sollten „die natürlichen, die Funktion des Körpers steuernden Gesetze" herausgefunden werden (ebd. S. 19). Zugleich gerieten die Gefahren sportlicher Überanstrengung in den Blick. Doping als ethisches oder moralisches Problem wurde dagegen nicht diskutiert. Für viele Ärzt*innen begann Doping erst dort, wo eine Gefährdung der Gesundheit zu befürchten war. Zudem galt die
Sportmedizin zunächst als Nebenbeschäftigung von wenigen interessierten Mediziner*innen. Die ersten Olympischen Spiele der Neuzeit 1896 in Athen bezeichnet Hoberman im Nachgang als „Beginn eines gigantischen biologischen Experiments" (ebd. S. 119).

Für die Debatten jener Zeit war eine entscheidende Frage, „ob man den sportlichen Ehrgeiz und Methoden, die diesem Ehrgeiz dienen könnten, einschränken sollte“ (ebd. S. 156). Neu eingebracht wurde dann, dass Pharmaka, „die zur Regeneration des Körpers des Athleten nach einem belastenden Wettkampf beitragen", Sinn machen würden (ebd. S. 167). Schon damals verstanden sich manche Mediziner*innen als Helfende beim Streben nach sportlicher Höchstleistung. Obwohl es noch keine Regeln und keine Testmethoden gab, war bei den meisten moralische Klarheit gegeben, dass man nicht alles machen darf, was möglich ist. Der wissenschaftliche Fortschritt vor allem in Medizin und Pharmazie eröffnete aber nach und nach ungeahnte Möglichkeiten der Leistungsmanipulation. Englische und amerikanische Zeitungen begannen, die Verwendung von Stimulantien als Betrug zu bezeichnen (Dimeo und Møller 2018, S. 22).

Beim zweiten Sportärztekongress 1924 in Berlin - eine Tagung des „Deutschen Reichskomitees für die wissenschaftliche Erforschung des Sportes und der Leibesübungen" - wurde eine Resolution gegen Doping verabschiedet, die in der Sportpraxis aber fast ohne Wirkung blieb (Singler 2012, S. 31). Beim dritten von der Chancengleichheit, Fairness und 


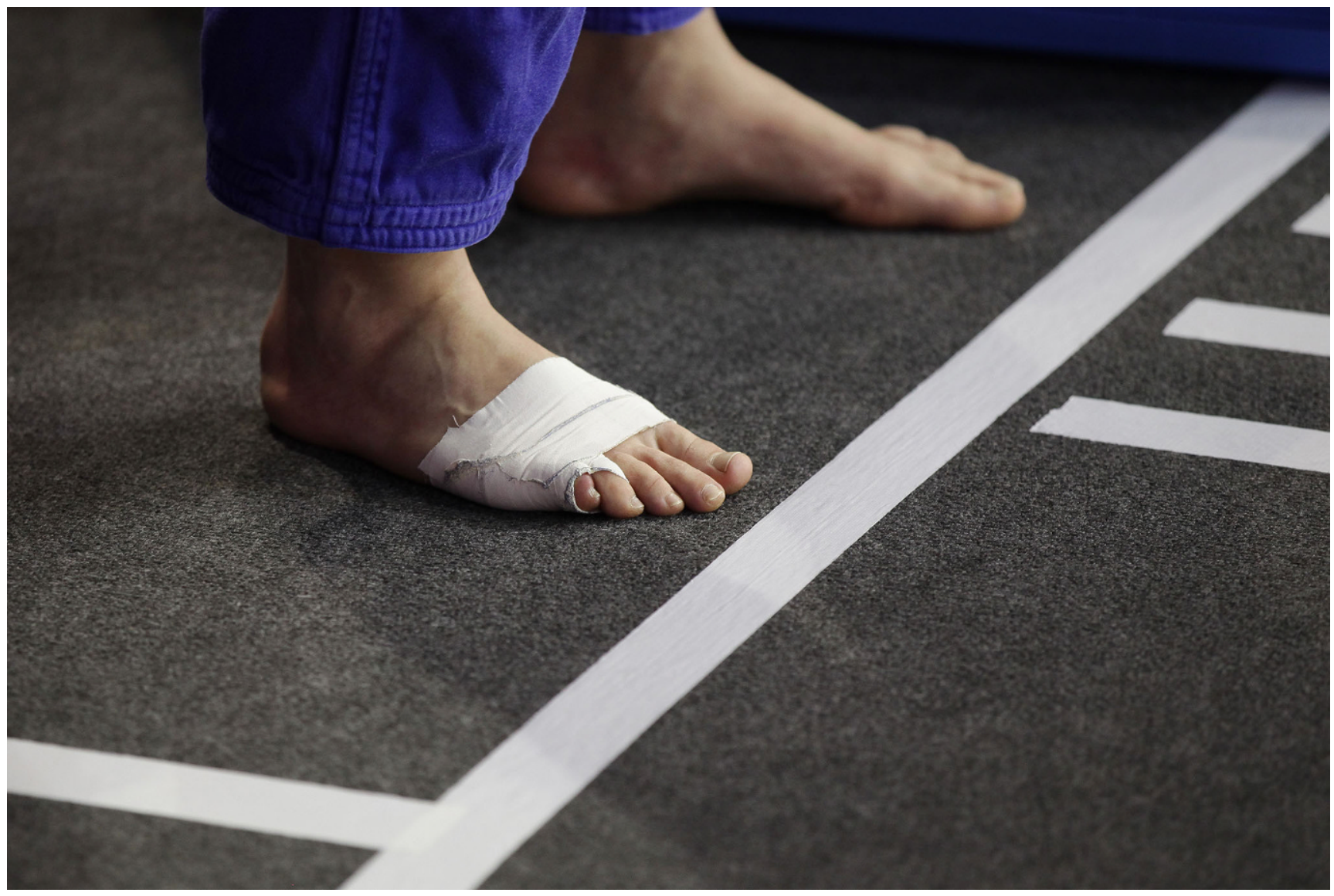

Abb. $1 \Delta$ Grenzlinie. Die bis heute anhaltende Auseinandersetzung für und gegen Doping betrifft die Frage: Gibt es für die Leistungssteigerung natürliche Grenzen und sind diese einzuhalten? Foto: Judo Magazin/Micha Neugebauer

Sportärztekongress 1927 wurde eine Unterscheidung zwischen Amateur*innen und Berufssportler*innen vorgeschlagen; bei Letzteren könne Doping angewendet werden, da der Schwerpunkt ihrer Sportausübung nicht auf sportlichem, sondern auf sozialem Erfolg liege, hieß es (Reinold 2016, S. 17). 1937 setzte der Schweizer Sportmediziner Gottfried Schönholzer „Berufssportler“ mit Spezialisten wie "Zirkusartisten“ gleich. Bei diesen sei der „eigentliche Sportgedanke, Gesundheit oder Fairness, sekundär geworden" (Aeschimann 2017, S. 73). Berufssportler*innen dürften zum Erreichen von Höchstleistungen alle verfügbaren Mittel einsetzen. Bei der 35. Sitzung des Internationalen Olympischen Komitees (IOC) im März 1938 in Kairo wurde vor der Verwendung von Drogen und künstlichen Stimulantien gewarnt - wer solche verwende, solle an Wettkämpfen von Amateur*innen nicht teilnehmen dürfen (Laure 2004, S. 163).
Zusammengefasst: Doping, die „neverending story“, begleitet den Spitzensport seit Anfang an.

\section{Bemühungen um den guten Ruf}

Nach dem Zweiten Weltkrieg gefährdeten Doping und damit verbundene Skandale zunehmend den "guten Ruf “ des Spitzensports. Die Dopingbekämpfung setzte allerdings erst ein, als in der Öffentlichkeit das positive Bild erschüttert wurde. Erst nach den Todesfällen des dänischen Radamateurs Knud Jensen bei den Olympischen Spielen 1960, des Straßenradweltmeisters Tom Simpson beim Anstieg zum Mont Ventoux bei der Tour de France 1965 oder des Boxers Jupp Elze 1968 folgten nach und nach Reaktionen, primär durch Ärzt*innen, die sich Sorgen über die Gesundheit der Sportler*innen machten.

Noch nicht gebremst von Regeln oder ethischen Überlegungen, experimentierten in jener Zeit vor allem Sport-Pro- fis mit Substanzen und Flüssigkeiten im Sinne eines Learning by Doing. Doping wurde zunächst einmal als Konsequenz des Ehrgeizes angesehen, die eigene Leistungsfähigkeit ohne Einschränkungen zu verbessern - was einer einseitigen erfolgsorientierten Auslegung des olympischen Mottos „citius, altius, fortius" entspricht. Fast zeitgleich entstanden Gegenbewegungen, zunächst nur in Form von Kritik am Doping, später in Form von Verboten. Die bis heute anhaltende Auseinandersetzung für und gegen Doping betrifft die Frage: Gibt es für die Leistungssteigerung natürliche Grenzen und sind diese einzuhalten (• Abb. 1)?

Der eigentliche Kampf gegen Doping begann erst Mitte der 1950er-Jahre. Mit der Verwendung von Amphetamin änderte sich innerhalb von zwei Jahrzehnten die Dopinglandschaft radikal. Bis Anfang der 1970er-Jahre waren Amphetamine die meistverwendeten Dopingmittel. Ergänzt und später abgelöst wurden sie ab etwa Ende der 1950er-Jahre durch 
anabole Steroide. Hinzu kam eine Abkehr vom eher unsystematischen Learning by Doing hin zu einem systematischeren, wissenschaftlich fundierten und oft von Sportmediziner ${ }^{*}$ innen unterstützten Doping.

Ein Beschleuniger der Dopingversuchung war der zunehmende Nationalismus im Sport. Mit Erfolgen im Sport konnte die Idee der Überlegenheit des eigenen Landes (und früher schon einer Rasse) in den Dienst der Politik gestellt werden. Neben Ärzt*innen, Trainer*innen und Funktionär*innen, die Doping von gesunden Athlet*innen als nicht verantwortbar ansahen, nahm die Zahl derer $\mathrm{zu}$, die entweder die Gabe von Dopingmitteln unter bestimmten Voraussetzungen als möglich (Leistungsmedizin) oder wegen der Erhaltung der Chancengleichheit als notwendig ansahen. Dopingregeln und -prävention zum Abbremsen der rasanten Entwicklung wurden erst dann als Aufgaben interessant, als Doping offensichtlich, das heißt für Zuschauer*innen erkennbar wurde - lange nach Beginn des Missbrauchs zum Beispiel von Stimulantien und Anabolika.

Ein ehrlicher Kampf gegen Doping hätte die internationale Konkurrenzfähigkeit damals ziemlich gefährdet. Bette und Schimank fassen den Kampf gegen Doping und das dopingbezogene Handeln des organisierten Sports und von Ärzt*innen in die Begriffe „brauchbare Illegalität“ (Steigerung der Zahl der Erfolge mit Hilfe von verbotenen Substanzen) und „symbolische Beschwichtigung" (Medaillen zur Rechtfertigung der Förderung durch Staat und Sponsoren bei gleichzeitiger Täuschung des Publikums, Bette \&Schimank 1995, S. 150 ff.). Franke hielt dazu 2012 fest: „Sie müssen einerseits so viel Kontrollen zulassen, dass ,Sauberkeit' als anstrebsamer Wert erkennbar wird, doch dürfen die Kontrollen nicht so wirksam sein, dass die Erfolgsmöglichkeiten im Vergleich zu allen anderen Teilnehmern deutlich reduziert werden“ (Franke 2012, S. 65).

Wie sollten Staat, Politik und organisierter Sport auf die wachsenden Probleme im Umgang mit Doping reagieren? Repression führt langfristig im Sinne Orwells zu einer Totalüberwachung von
Sportler*innen. Prävention mit dem Ziel, dass mündige und mental starke Sportler*innen zur Dopingversuchung Nein sagen und sich der Karriereengführung in die Dopingfalle (Bette/Schimank 1995, S. 110 f.) entziehen können, bringt höchstwahrscheinlich Leistungseinbußen und damit verbunden eine geringere Förderung durch Staat und Sponsoren mit sich. Sport und Politik waren und sind damit konfrontiert, dass die zunehmende Bedeutung des Leistungssports $\mathrm{zu}$ einer permanenten Suche nach „Abkürzungen“ auf dem Weg zu Erfolgen führte. Als Ausweg galt die von Bette und Schimank angedeutete Doppelstrategie mit der Kombination von brauchbarer Illegalität und symbolischer Beschwichtigung. Öffentliche Empörung über bekannt gewordene Dopingfälle erzeugte Druck und verstärkte die Angst um den guten Ruf des Spitzensports.

Zusammengefasst: Dopinghandeln war zunächst ein Learning by Doing von wenigen. Ein Kampf dagegen begann nur sehr langsam. Erst als Folgen auch für die Öffentlichkeit sichtbar wurden, wurde er intensiviert, aber zunächst nur als Repression. Ein intensiver Kampf hätte Folgen für die Erfolgsbilanz und Mittelzuwendungen von Staat und Sponsoren gehabt. Der organisierte Sport wählte den Weg der Kombination von brauchbarer Illegalität und symbolischer Beschwichtigung. Gefährlich ist dabei vor allem der Nationalismus auf den verschiedenen Ebenen (Staat, Region, Kommune).

\section{Entwicklung von Doping- mentalität}

Wichtiger als die Bemühungen des organisierten Sports und des Staats um sauberen Sport ist die Frage, ob und wie sich eine Dopingmentalität als Voraussetzung für den Weg in die Dopingfalle (Bette/ Schimank) entwickeln kann. Dies hängt wesentlich vom Vorbild der Eltern und des$^{\star} r$ Trainer ${ }^{*}$ in ab. Sind diese eindeutig gegen den Konsum von illegalen und legalen Mitteln, ist die Wahrscheinlichkeit groß, dass keine Dopingmentalität entsteht und der Weg in die Dopingfalle vermieden wird. Vom Vorbild und der Gestaltung der Beziehungsebenehängt es $\mathrm{ab}$, ob sich Sportler*innen mündig und selbstbestimmt für oder gegen deviantes Verhalten entscheiden können.

Eine negative Gestaltung der Beziehungsebene kann auch für Erfolge sorgen und wird von einzelnen Trainern bevorzugt, wie zum Beispiel der berüchtigte Ausspruch des Sprint-Bundestrainers Wolfgang Thiele zeigt: „Das Verhältnis Trainer/Sportlerin kann erst dann richtig leistungsfördernd sein, wenn es in der Grundstruktur dem des Zuhälters zur Prostituierten entspricht" (Treutlein 2011, S. 136). Eine solche Gestaltung forciert eine weitgehende bis völlige Unterordnung, vor allem von Mädchen und Frauen - das absolute Gegenteil der von der dsj verfolgten Ziele zur positiven Entwicklung der Persönlichkeit.

Dopingmentalität entwickelt sich weiterhin bei der Suche nach "Abkürzungen“ auf dem Weg zu angestrebten und nicht selten mit normalen Mitteln wie Training und gesunder Lebensführung nicht erreichbaren Leistungszielen. Hierfür werden zunehmend Nahrungsergänzungs- und Schmerzmittel verwendet - Mittel, die nicht auf den Verbotslisten von WADA und NADA stehen (- Abb. 2). Solche legalen Mittel werden systematisch gesucht. Ihr Konsum ist selten leistungsfördernd, kann aber aus mehreren Gründen problematisch sein: Zum einen kann die Interaktion mehrerer zeitgleich verwendeter Mittel $\mathrm{zu}$ schädlichen Nebenwirkungen führen. Zum anderen kann der Konsum dieser legalen Mittel durch die Gewöhnung an eine Medikamentenverwendung eine Stufe hin zur Entwicklung von Dopingmentalität sein - ein großes Risiko für die Gesundheit von jungen Leistungssportler*innen und für den sauberen Sport (Deutsche Sportjugend 2018). Schmerzmittel werden schon seit Jahrzehnten vor allem in Kontaktsportarten wie Fußball und Handball sowie im Ausdauersport konsumiert. Das frühzeitige Gewöhnen an Pilleneinnahmen senkt die Hemmschwelle des Übergangs zum Konsum verbotener Präparate. Pillen zur Leistungssteigerung einzunehmen, ist ein großer Schritt auf den nachfolgenden Stufen der Versuchung beziehungsweise in die Dopingmentalität.

Trotz aller Erkenntnisse zur Dopinggeschichte und Dopingskandalen der 


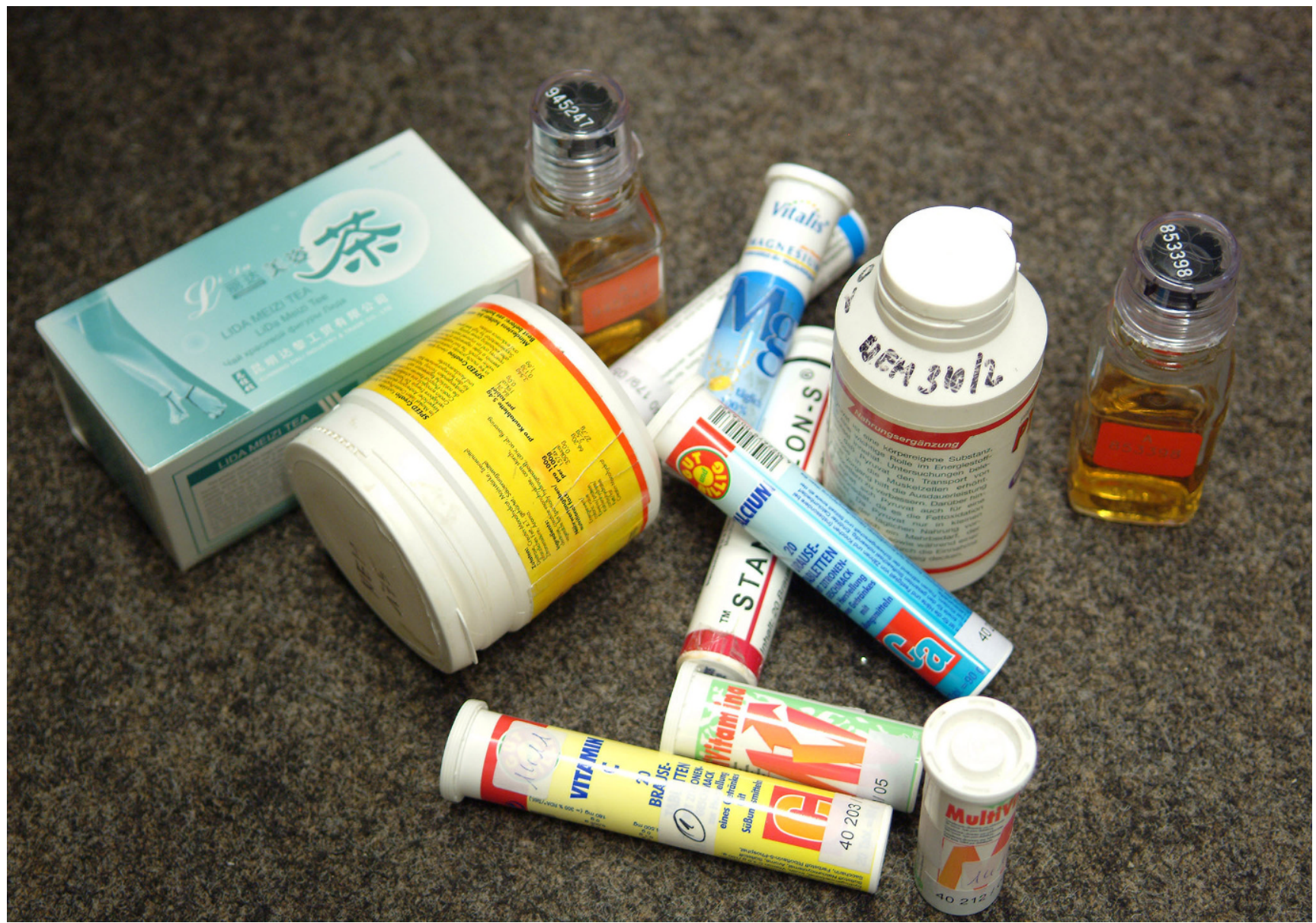

Abb. 2 \ Dopingmentalität entwickelt sich bei der Suche nach „Abkürzungen“ auf dem Weg zu Leistungszielen. Hierfür werden zunehmend Nahrungsergänzungs- und Schmerzmittel verwendet - Mittel, die nicht auf den Verbotslisten stehen. Foto: LSB NRW/Andrea Bowinkelmann

vergangenen Jahrzehnte (zum Beispiel zum Dopingfall Ben Johnson bei den Olympischen Spielen 1988, zum Doping des Radteams Festina bei der Tour de France 1998 und des Teams Telekom 2007) ist das Problembewusstsein für die Notwendigkeit einer sinnvollen Dopingprävention in der Öffentlichkeit und bei nicht wenigen Vereinen und Verbänden nach wie vor nicht ausreichend (• Abb. 3). Dafür gibt es unter anderem folgende Gründe:

- „Die Einschläferungsbotschaften des Freiburger Sportmedizinchefs Prof. Dr. Joseph Keul u. a.m., dass es eigentlich kaum Doping gäbe und zudem Doping mit anabolen Steroiden unwirksam sei. Sie förderten den naiven Glauben der Öffentlichkeit und vieler engagierter Ehrenamtler im Sport an den ,sauberen' bundes- deutschen Sport" (Krüger et al. 2014, S. 207).

- Die als Dopingprävention angepriesene Vertuschungsrhetorik wurde von Sportler ${ }^{\star}$ innen als solche erkannt und nicht ernst genommen (Singler 2011, S. 51).

- Überforderungsempfinden von Verantwortlichen im Sport: Sie hätten schon so viele Aufgaben, jetzt sollten sie sich auch noch um die Dopingbekämpfung kümmern: Die Dopingproblematik sei auf dem von ihnen betreuten Leistungsniveau nicht relevant, der Kampf gegen Doping übertrieben, für Dopingprävention bedürfe es externer Hilfe (Singler 2011, S. 83 ff.).

- Olympische Spiele und internationale Meisterschaften wurden im Lauf der Jahrzehnte zunehmend zu zivilen „Kriegsschauplätzen“ für das Aus- leben nationalistischer Interessen, sichtbar am überhöhten Stellenwert von Medaillenspiegeln. Dopingprävention konnte da nur stören. Wenn Problembewusstsein für die Notwendigkeit von Dopingprävention fehlt, taucht die Frage auf, warum dann ausgerechnet Deutschland Vorreiter der Dopingprävention werden soll?

Der Münsteraner Sporthistoriker Michael Krüger und der Sportjournalist Ralf Meutgens bringen die Problematik der Zielsetzung von Spitzensport auf den Punkt: In seinem Artikel „The winner takes it all“ sieht Krüger den Spitzensport im Dilemma zwischen wertorientiertem Leistungsstreben einerseits und Siegen um jeden Preis andererseits. Dabei hatte der Erneuerer der Olympischen Spiele, Pierre de Coubertin, die olympische Idee als Erziehungsidee verstanden und 


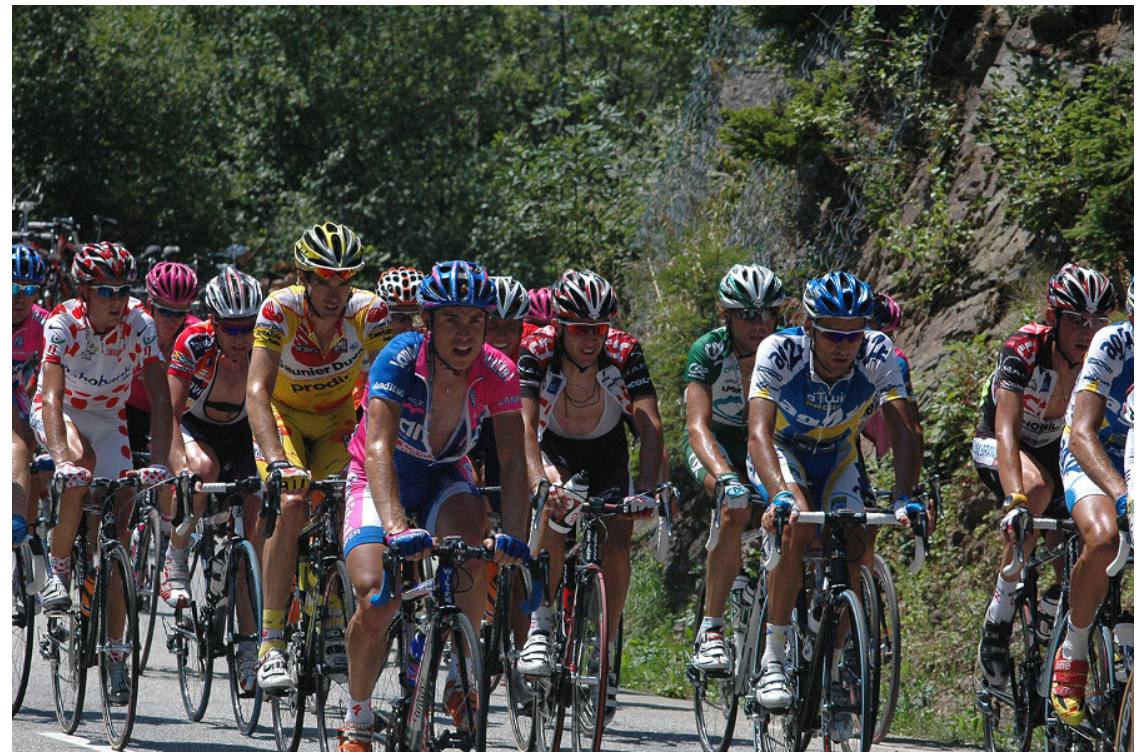

Abb. $3 \Delta$ Trotz Dopingskandalen wie bei der Tour de France (hier das Fahrerfeld im Jahr 2006) ist das Problembewusstsein für die Notwendigkeit einer sinnvollen Dopingprävention nach wie vor nicht ausreichend. Foto: Pixelio.de/Hans-Peter Reichartz

damit den Schwerpunkt auf die Wertorientierung gelegt (Krüger 2013, S. 23-33). Anspruch und Wirklichkeit klafften im Laufe der folgenden Jahrzehnte immer weiter auseinander. Meutgens schreibt im Editorial des Schwerpunkthefts „Dopingprävention“ der Zeitschrift „Doping“ zum genannten Dilemma: „Der Irrglaube an ein unbegrenztes Wachstum, an immer neue Bestleistungen und an immer mehr Medaillen, das Streben nach Perfektion scheint derart in den Köpfen vieler Zeitgenossen einbetoniert $\mathrm{zu}$ sein wie der Wunsch nach der Top-Figur, dem bestbezahlten Job, der ständigen Erreichbarkeit und Leistungsfähigkeit oder ähnlichen Auswüchsen. Der Selbstoptimierungswahn kennt keine Grenzen (Meutgens 2018, S. 57).“

Es ist an der Zeit, die bisher weitgehend unterlassene Zieldiskussion für den deutschen Spitzensport offen zu führen. Nach Jean-Pierre de Mondenard (von 1974 bis 1977 Rennarzt bei der Tour de France) müsste das olympische Motto "citius, fortius, altius" ersetzt werden, weil es Fehlentwicklungen des modernen Sports begünstigt. De Mondenard schlägt beispielsweise vor: „Immer weniger Betrug, kein Doping mehr, das Geld darf keine Rolle spielen“ (De Mondenard 1996, S. 16).
Zusammengefasst: Entscheidend für die Entwicklung von Dopingmentalität sind Vorbilder und die Gestaltung der Beziehungsebene. Eine frühzeitige Gewöhnung an den Konsum von Pillen kann die Entwicklung von Dopingmentalität beschleunigen. Das Problembewusstsein ist bisher nicht ausreichend entwickelt. Nötig wäre eine gründliche Diskussion der Zielsetzung von Spitzensport. Eventuell ist auch die Veränderung des olympischen Mottos "citius, altius, fortius" notwendig, da die vorherrschende einseitige erfolgsorientierte Auslegung Fehlentwicklung im Leistungs- und Spitzensport begünstigt.

\section{Präventionsansätze}

In einer engen Auslegung der Begriffe "Aufklärung" und „Dopingprävention" wird darunter nur das Informieren über Dopingregeln, Kontrollen und $\mathrm{Be}$ strafung verstanden. In einer weiten Auslegung ist unter „Aufklärung“ die Überwindung von Unmündigkeit zu verstehen, ein Aufruf zum Nutzen des eigenen Verstands: Mündige Athlet ${ }^{\star}$ innen sollen sich bewusst für oder gegen Doping entscheiden können und Verantwortung für die Folgen ihrer Entscheidung übernehmen. Bei der Dopingprävention wird zudem im Wesentlichen zwischen Verhal- tens- (das Subjekt als Adressat) und Verhältnisprävention (die Strukturen betreffend) unterschieden.

Bisher diskutierte Ansätze der Dopingprävention sind:

1. Abschreckungsansatz (repressiver

Bereich, Kontrollen und Bestrafung:

„Wenn du dopst, spielst du mit deinem Leben").

2. Verheimlichung von Wissen und Negierung leistungssteigernder Effekte (Verweise auf mögliche Placebo-Effekte).

3. Aufklärungskonzept (Idealvorstellung: Von der Informationsvermittlung zur Einstellungs- und Verhaltensänderung).

4. Risikofaktorenkonzept (Risikobewusstsein schärfen, Wissen vermitteln zu Wirkungen und Nebenwirkungen sowie zu Risiken erhöhenden und vermindernden Faktoren).

5. Salutogeneseansatz (Gesundheitsorientierung, Förderung von gesundheitsorientierten Prozessen).

6. Kompetenzorientierte Prävention und Gesundheitsförderung (Fähigkeiten und Fertigkeiten zur Bewältigung von Aufgaben und Problemstellungen erlernen).

7. Settingansatz (systemorientierte Maßnahmen, Einbeziehung der Lebenswelt, Doping ablehnende Strukturen, Schaffung gesundheitsförderlicher Lebenswelten).

Die pathogenetische (repressive) Orientierung der Dopingbekämpfung (Punkte 1,2 und 4) reicht ebenso wenig aus wie eine Aufklärung zu Regeln, Kontrollen und Bestrafung (Punkt 3). Nur die Kombination von Aufklärung im engen mit jener im weiten Sinne (mit salutogenetischer Orientierung) dürfte einigermaßen erfolgversprechend sein.

Ein Fehler der Ansätze 1 bis 4 ist zum einen die Erwartung, dass das Umsetzen von Wissen in Handeln automatisch eine Folge des Vermittelns und Anhäufens von Wissen sei; doch das bleibt meist aus (funktionaler Ansatz, Treutlein et al. 1996). Wer Mündigkeit, Reflexion und Verantwortung anstrebt, muss zu einem intentionalen Ansatz übergehen, für den dann aber auch mehr Zeit und didaktisches Können zur Verfügung gestellt 
werden muss als nur ein zweistündiger Vortrag. Dopingprävention (im weiten Sinne) ist eine Investition in die Zukunft und müsste mindestens ebenso gut finanziell und personell ausgestattet werden wie der ganze Dopingkontrollapparat.

Auffallend ist, dass es für die Konzepte 1 bis 4 schon genügend Materialien gibt, nicht aber für die anderen Ansätze. Im Gegensatz zu Frankreich fehlen ausgebildete Dopingpräventionsfachleute; ihre Ausbildung wurde bisher weder von NADA noch von DOSB in Angriffgenommen. Der Ansatz der dsj mit ,Juniorbotschafter*innen für Dopingprävention" ist zwar sehr vielversprechend. Man kann aber von den jungen Leuten, die neben der Dopingprävention auch Aufgaben wie Studium, Beruf und ehrenamtliches Engagement zu bewältigen haben, nicht erwarten, dass sie sich im Selbststudium die ganze Breite der Dopingpräventionsproblematik erarbeiten. Es bringt schon viel, wenn sie ihre durch Studium und Beruf schon vorhandenen Schwerpunkte in die Präventionsarbeit einbringen.

In den beiden vergangenen Jahrzehnten haben sich für Deutschland vor allem zwei wesentliche Präventionsstrategien herausgebildet, mit schwerpunktmäßig unterschiedlichen Zielen und Strategien:

Präventionsstrategie 1, einer engen Auslegung von Aufklärung folgend: Hier wird das Ziel verfolgt, Sportler*innen vor einem positiven Dopingtest zu schützen durch Informationen über die Dopingregeln (WADA, NADA), den Ablauf von Dopingkontrollen, den Analysevorgang und Bestrafungsmöglichkeiten. Diese reduzierte Form von Aufklärung kann dazu führen, dass Sportler*innen nach Möglichkeiten suchen (oder wissen, was sie tun müssen), um erfolgreich dopen zu können und trotzdem in einer Kontrolle nicht positiv kontrolliert zu werden. Dem Kontroll-/Testbereich wird angesichts dessen, dass nachgewiesenermaBen nur ein geringer Teil der Dopenden überführt wird, ein unangemessen hoher Stellenwert eingeräumt. Als Grundlage für die Bearbeitung der Thematik mit der Präventionsstrategie 2 sind solche Basisinformationen allerdings wichtig und notwendig.
Präventionsstrategie 2 auf der Basis eines weiten Begriffs von Aufklärung: Sie beinhaltet Zielsetzungen wie „Impulse zur Mündigkeitsentwicklung “, ,Benutzen des Verstands“, „Selbstbestimmung“ und „Befreiung aus der Unmündigkeit“ dies sind Schwerpunkte der Präventionsarbeit der dsj. Sie sieht sich der gegenwärtigen und zukünftigen Entwicklung von jungen Sportler*innen in Sport und Alltag sowie des Sportsystems verpflichtet. Am erfolgversprechendsten hat sich eine Kombination von Top-downund Bottom-up-Strategie herausgestellt. Bei dsj-Seminaren für zukünftige Juniorbotschafter*innen für Dopingprävention sind Themen wichtig wie gesunde Lebensführung, mentale Stärkung, das Unterbinden nationalistischer Tendenzen oder Spitzensportdoping als „Negativvorbild“ für Alltagsdoping (Resilienz und Settingansatz).

Statt einer Konzentration auf eine pathogenetische Sichtweise des Problems versucht die dsj, in ihre Kombination von Top-down- und Bottom-up-Strategie eine salutogenetische Sichtweise einzubringen. Dopingmentalität entsteht sehr früh, vom Kindesalter an. Entsprechend früh müssen Anstrengungen zum Aufbau einer Anti-Betrugshaltung und zu einer gesunden Lebensführung beginnen. Deshalb muss Dopingprävention in der Kindheit und Jugend anfangen, unspezifisch bereits bei Kindern (Schwerpunkt gesunde Lebensführung), spezifisch eher nach der Pubertät, wenn die Fähigkeit zum Reflektieren ausreichend entwickelt ist. Werden junge Sportler*innen in einem Umfeld groß, in dem einseitig der Schwerpunkt auf Leistungssteigerung gelegt wird, haben Doper*innen später weder ein schlechtes Gewissen, noch Anlass oder Motivation, ihr Verhalten grundlegend zu hinterfragen oder gar zu verändern. Elternarbeit muss daher eine wichtige Forderung sein.

Besondere Schwerpunkte im dsj-Präventionsansatz liegen aufder Bottom-upStrategie, einer interaktiven Vorgehensweise und Peer-Education. Diese hat das Ziel, dass junge Leute mit Problembewusstsein (Juniorbotschafter*innen für Dopingprävention) ihre Peers (Vereinsoder Klassenkamerad ${ }^{*}$ innen, Jugendgruppe und andere in ähnlichem Alter) $\mathrm{zu}$ beeinflussen versuchen (Vorbildwirkung, Lernen von Gleichaltrigen, mit Gleichaltrigen und zugleich Lernen durch Lehren). Tatsächlich wäre hierzu eine ausführliche Schulung zum Beispiel der Juniorbotschafter ${ }^{*}$ innen sinnvoll und notwendig. Angesichts geringer finanzieller und personeller Mittel liegt der Schwerpunkt bisher auf einer relativ kurzen Intervention (zwei zweitägige Seminare). Es hat sich aber gezeigt, dass schon eine so kurze Schulung wesentliche Impulse für eine Peer-Tätigkeit und für ehrenamtliches Engagement setzen kann. Peers mit Problembewusstsein können so zu Vorbildern werden, die zur Nachahmung anregen. Es gibt genügend Beispiele von Juniorbotschafter*innen, die auf Grundlage der in den dsj-Seminaren vermittelten Impulse begannen, für die Dopingprävention in ihren Verbänden Verantwortung zu übernehmen und diese aktiv zu gestalten.

Bei der Vorgehensweise sind vor allem folgende Prinzipien erfolgreich und zu beachten:

- Jede Präsenzveranstaltung ist effektiver als die Beschäftigung mit schriftlichen Materialien und/oder mit Angeboten im Internet (zum Beispiel der NADA [https://www. gemeinsam-gegen-doping.de]) oder der dsj (über Suche Dopingprävention unter https://www.dsj.de/nc/ publikationen). Beispielsweise wird das E-Learning-Programm des Nationalen Olympischen Komitees in Frankreich kaum genutzt (Patrick Magaloff: „Qu'est-ce que le programme antidopage du CNOSF?", Cycling4Fans 2016).

- Eine interaktive Vorgehensweise bei Präsenzveranstaltungen ist effektiver als Beiträge in Vortragsform (Frontalunterricht).

- Bei den verschiedenen Programmpunkten bei dsj-Seminaren sollten nach Möglichkeit bereits ernannte dsj-Juniorbotschafter*innen, die sich zu einzelnen Punkten zu Expert ${ }^{\star}$ innen weiterentwickelt haben, als Referent ${ }^{*}$ innen und/oder Gestalter*innen eingesetzt werden (Lernen durch Lehren, Vorbildwirkung).

- Der Einsatz von Zeitzeug*innen, die über ihre Erlebnisse und Er- 
fahrungen berichten, wie Claudia Lepping (deutsche LeichtathletikJuniorenrekordlerin über $200 \mathrm{~m}$; sie hatte früh eine Antidopinginitiative gegründet und war Whistleblowerin zum Doping beim „HammerModell“ des Dopingtrainers Jochen Spilker), Henner Misersky (mehrfacher DDR-Meister über 3000-MeterHindernis, Dopingverweigerer und -gegner, Vater der Biathlon-Olympiasiegerin von 1992, Antje Misersky), Sven Laforce (Dopingkontrolleur bei sechs Olympischen Spielen und bei der Fußball-Weltmeisterschaft 2006) oder Simon Krivec (Bericht über seine Forschungsarbeit „Die Anwendung von anabolen-androgenen Steroiden in der Bundesrepublik Deutschland in den Jahren 1960 bis 1988 unter besonderer Berücksichtigung der Leichtathletik“) führen zur Vertiefung des Problembewusstseins der Teilnehmer*innen.

- Berichte von Juniorbotschafter ${ }^{\star}$ innen über ihre ehrenamtlichen Aktivitäten in Landes- und Bundessportverbänden wie durch Kaja Schröder (Antidopingbeauftragte des Landesverbands Schleswig-Holstein der Deutschen Triathlon-Union), Lukas Monnerjahn (hat Ausbildungsmaterialien zur Dopingprävention für den DJK-Sportverband erarbeitet), Helen Diederich/Lea Saur (projektbegleitend zum Mannheim-Marathon), Jasmin Richter (Projekte in der Deutschen Taekwondo Union), Henrietta Weinberg (Vorstandsmitglied dsj), Denise Brachert (Jugendvorstand des Deutschen Fechter-Bunds), Hans-Peter Gratz (Disziplinchef Schwimmen im Allgemeinen Deutschen Hochschulsportverband) oder Moritz Belman/Florian Ellmann (Projekte im Deutschen Judo-Bund) und andere mehr erhöhen die Motivation für eigene Aktivitäten. Die Berichterstatter*innen sind zugleich Vorbilder für die Seminarteilnehmer ${ }^{\star}$ innen.

Trotz Widerständen und Rückschlägen sollten die Verfechter*innen eines ehrlichen Sports und einer gesunden Lebensführung sich immer wieder das Bild des Sisyphos vor Augen führen: Sämtliche
Maßnahmen zur Dopingprävention stehen sinngemäß dafür, den Stein Dopingprävention mit großer Kraftanstrengung nach oben - zum Gipfel sauberer Sport zu rollen. Das Ziel ist nie vollständig erreichbar - und muss von jeder folgenden Generation jugendlicher Sportler*innen von neuem angegangen werden.

Zusammengefasst: Aufklärung (im reduzierten Sinne, Schwerpunkte Kontrolle und Bestrafung) und eine pathogenetische Orientierung waren beim Abbremsen und Verhindern von Doping bisher wenig erfolgreich. Größere Erfolgschancen haben salutogenetische Ansätze beziehungsweise solche, wie sie die dsj mit dem Aktivieren von Juniorbotschafter*innen für Dopingprävention verfolgt (Schwerpunkte Reflexions- und Entscheidungsfähigkeit, Peer-Education, Bottom-up-Strategie).

\section{Ausblick}

Doping und Dopingprävention sind zentrale Herausforderungen des modernen Leistungssports. Eine Hoffnung für den sauberen Sport sind die Aktivitäten der "Aufrechten“ wie zum Beispiel Brigitte Berendonk, Werner Franke und Elk Franke, Hans-Jörg Kofink, Karl-Heinrich-Bette beziehungsweise die Aktivitäten der nachfolgenden Generationen (zum Beispiel Claudia Lepping, Ralf Meutgens). Eine Hoffnung auf Entstehen und Weiterentwicklung einer Graswurzelbewegung zugunsten eines sauberen Sports versprechen auch die von der dsj zu Juniorbotschafter ${ }^{\star}$ innen für Dopingprävention ernannten jungen Menschen. Sie müssen unter anderem medialen Druck zugunsten einer salutogenetisch orientierten Dopingprävention erzeugen!

\section{Korrespondenzadresse}

\section{Prof. Dr. Gerhard Treutlein}

Zentrum für Dopingprävention, Pädagogische Hochschule Heidelberg

Heidelberg, Deutschland

treutlein@ph-heidelberg.de

Prof. Dr. Gerhard Treutlein ist pensionierter Professor für Sportpädagogik der Pädagogischen Hochschule Heidelberg. Seit Oktober 2007 leitete er dort das Zentrum für Dopingprävention, das zusammen mit der Deutschen Sportjugend Materialien und Seminarfor- men für Dopingprävention erarbeitet hat. Vor allem für seine Doping-Präventionsarbeit erhielt er 2016 den Ethikpreis des Deutschen Olympischen Sportbunds.

Funding. Open Access funding enabled and organized by Projekt DEAL.

Open Access. Dieser Artikel wird unter der Creative Commons Namensnennung 4.0 International Lizenz veröffentlicht, welche die Nutzung, Vervielfältigung, Bearbeitung, Verbreitung und Wiedergabe in jeglichem Medium und Format erlaubt, sofern Sie den/die ursprünglichen Autor(en) und die Quelle ordnungsgemäß nennen, einen Link zur Creative Commons Lizenz beifügen und angeben, ob Änderungen vorgenommen wurden.

Die in diesem Artikel enthaltenen Bilder und sonstiges Drittmaterial unterliegen ebenfalls der genannten Creative Commons Lizenz, sofern sich aus der Abbildungslegende nichts anderes ergibt. Sofern das betreffende Material nicht unter der genannten Creative Commons Lizenz steht und die betreffende Handlung nicht nach gesetzlichen Vorschriften erlaubt ist, ist für die oben aufgeführten Weiterverwendungen des Materials die Einwilligung des jeweiligen Rechteinhabers einzuholen.

Weitere Details zur Lizenz entnehmen Sie bitte der Lizenzinformation auf http://creativecommons.org/ licenses/by/4.0/deed.de.

\section{Literatur}

\section{Verwendete Literatur}

Aeschimann, W. (2017). Erste Dopingdiskussionen in der Schweiz an den Beispielen Coramin, Cardiazol und Pervitin (1925-1945). In STG/RSH/RSS 67, 201, Nr. 1 (S. 59-78).

Bette, K.-H. \& Schimank, U. (1995): Doping im Hochleistungssport: Anpassung durch Abweichung. Frankfurt am Main: Suhrkamp.

Cycling4Fans (2016). Anmerkungen von Prof. Dr. Gerhard Treutlein, 16. französische Tagung Doping und Dopingprävention - Paris, 15./16. April 2016. https://www.cycling4fans.de/index. php?id=6354. Zugegriffen: 26. Okt. 2020.

De Montenard, J.-P. (1996): Le dopage aux Jeu Olym pique - La triche récompensée. Paris: edition amphora.

Deutsche Sportjugend (2018). Wie begegnen wir der Entwicklung von Dopingmentalität? https://www.dsj.de/fileadmin/user_upload/ Mediencenter/Publikationen/Downloads/dsj_ dopingmentalitaet2018.pdf. Zugegriffen: 5 . Febr. 2021.

Dimeo, P., \& Møller, V. (2018). Anti-doping crises in sport. Causes, consequencies, solutions. London, New York: Routledge.

Franke, E. (2012). Zwischen individueller Freiheit und institutioneller Verantwortung - zu Grenzen des Rechts und Möglichkeiten der Moral im Kampf gegen Doping. In G. Spitzer \& E. Franke (Hrsg.), Sport, Doping und Enhancement(S. 39-74). Köln Sportverlag Strauß.

Hoberman, J. (1994). Sterbliche Maschinen. Doping und die Unmenschlichkeit des Hochleistungssports. Aachen: Meyer \& Meyer.

Krüger, M. (2013). "The winner takes it all“ - Sport zwischen wertorientiertem Leistungsstreben und Siegen um jeden Preis. In A. R. Hofmann \& 


\section{Fachbeitrag}

M. Krüger (Hrsg.), Olympia als Bildungsidee. Beiträge zur olympischen Geschichte und Pädagogik (S. 23-33). Heidelberg: SpringerVS.

Krüger, M., \& Becker Nielsen Reinold, C. S. M. (2014). Doping und Anti-Doping in der Bundesrepublik Deutschland 1950-2007. Genese - Strukturen Politik. Hildesheim: Arete.

Laure, P. (2004): Histoire du dopage et des conduites dopantes: Les alchimiste des la performance. Paris:Vuibert.

Meutgens, R. (2018). Sind wir noch zu retten? - Ein Kommentar.Zeitschrift Doping, 5, 57.

Reinold, M. (2016): Doping als Konstruktion. Eine Kulturgeschichte der Anti-Doping-Politik. Bielefeld: transcript Verlag.

Singler, A. (2011). Dopingprävention - Anspruch und Wirklichkeit. Aachen: Shaker.

Singler, A.\& Treutlein, G. (2012): Doping im Spitzensport Sportwissenschaftliche Annalysen zur nationalen und internationalen Leistungsentwicklung. Aachen:Meyer und Meyer.

Treutlein, G. (2011). Zum Problem der Abhängigkeit und Fremdbestimmung in der Frauen-Leichtathletik. In F. Dannenmann \& R. A. Meutgens Singler (Hrsg.), Sportpädagogik als humanistische Herausforderung (S. 135-142). Aachen: Shaker.

Treutlein, G., Janalik, H., \& Hanke, U. (1996). Wie Sportlehrer wahrnehmen, denken, fühlen und handeln (4. Aufl.). Köln: Sport und Buch Strauß.

\section{Weiterführende Literatur}

Bette, K.-H., \& Schimank, U. (2006). Doping im Hochleistungssport. Anpassung durch Abweichung (2. Aufl.). Frankfurta.M.: Suhrkamp.

Brissonneau, Ch (2006). Die "préparation biologique“ in Frankreich. Subjektive Definitionen der Sportmedizin als Türöffner für neue Formen des Dopings. In W. Knörzer \& G. G. Spitzer Treutlein (Hrsg.), Dopingprävention in Europa (S. 107-111). Aachen: Meyer \& Meyer.

Laure, P. (2018). Die Prävention von Doping und Dopingverhalten aufwerten! Zeitschrift Doping, 5, 102-107.

Lautenbach, P., Becker, J., \& Kilian, D. (2018). Peerto-Peer. Das Juniorbotschafter/innen-System Dopingprävention der Deutschen Sportjugend. Zeitschrift Doping, 5, 68-73.

Singler, A., \& Treutlein, G. (2010). Doping - von der Analyse zur Prävention. Vorbeugung gegen abweichendes Verhalten in pädagogischem und soziologischem Zugang (2. Aufl.). Aachen: Meyer \& Meyer.

Singler, A., \& Treutlein, G. (2013). Historische und soziologische Aspekte abweichenden Verhaltens im Spitzensport. In A. R. Hofmann \& M. Krüger (Hrsg.), Olympia als Bildungsidee. Beiträge zur olympischen Geschichte und Pädagogik (S. 157-183). Wiesbaden:Springer.

Treutlein, G. (2002). Pädagogisch orientierte DopingPrävention - eine verdrängte Aufgabe des deutschen Sports. In A. Horn (Hrsg.), Sport macht Schule - Kinder stark machen in Schule und Verein. Fachkongress in Schwäbisch Gmünd am 4./5. April 2003. Gmündner Hochschulreihe, (Bd. 23, S. 61-70)

Treutlein, G. (2018).Zukunftsweisende Dopingprävention. Dopingmentalität als Schwerpunktthema moderner Dopingprävention.Zeitschrift Doping, 5,116 . 